Gut, 1971, 12, 639-645

\title{
Gastric ulcer and gastritis
}

\author{
M. W. L. GEAR ${ }^{1}$, S. C. TRUELOVE, AND R. WHITEHEAD \\ From the Nuffield Department of Clinical Medicine and The Department of Morbid Anatomy, Radcliffe \\ Infirmary, Oxford
}

SUMMARY The gastritis associated with chronic gastric ulcer has been studied by means of biopsy specimens taken under vision through a fibreoptic gastroscope from four standard sites in the stomach. Observations have been made in patients with untreated gastric ulcer and also in patients after medical or surgical treatment of the ulcer. The gastritis is usually widespread in chronic peptic ulcer of the body of the stomach, whereas it is commonly more localized in chronic prepyloric ulcer. Superficial or atrophic gastritis has been found to persist or even worsen after healing of the ulcer, whether the treatment was medical or surgical. This finding suggests that gastritis is the basic disease process and that gastric ulceration is a secondary phenomenon.

Gastric ulcer is frequently associated with chronic gastritis but the relationship between the two is controversial. On the one hand, there have been studies suggesting that the gastritis is the primary change with the gastric ulcer supervening (Faber, 1935; Hebbel, 1943; Magnus, 1946; Hebbel, 1949; Magnus, 1952). On the other hand, there are studies which suggest that the ulcer is the primary lesion and that the gastritis is a zonal change around it (Schindler, 1947; Palmer, 1954; Joske, Finckh, and Wood, 1955).

The methods employed in all these studies are open to criticism. Necropsy studies, even when performed with fixation immediately after death, are not ideal because of agonal changes and autolysis. Gastrectomy specimens suffer from two disadvantages: first, only the distal segment of the stomach is available for examination and, secondly, operative manoeuvres have been shown to cause inflammatory changes in the gastric mucosa (Schindler, Necheles, and Gold, 1939; Sanders and Mecray, 1941). Peroral biopsy specimens obtained by a Wood's tube or similar instrument suffer from the severe disadvantage that there is little control over the location of the biopsy site. Radiological examination has shown that the biopsy tube usually runs from the oesophagus to the greater curve of the stomach, so that the biopsy specimen comes from the greater curve or close to it. Pyloric mucosa is seldom obtained (Macdonald and Rubin, 1967).

The most recent fibreoptic gastroscopes incor-

Received for publication 18 May 1971.

'Present address: Gloucestershire Royal Hospital, Southgate Street, Gloucester. porate facilities for taking multiple biopsy specimens under direct vision from any part of the stomach. Such an instrument provides the means of studying the relationship between gastritis and gastric ulcer in a manner which eliminates most of the objections to earlier methods of study. Previous methods of gastroscopic biopsy, such as those of Kenamore (1940), Benedict (1948), and Hancock and Shiner (1958), suffered from various disadvantages which prevented their general adoption. In the present series we have used the Olympus GFB gastroscope to make a systematic study of the gastritis associated with gastric ulcer.

\section{Patients}

Ninety-five patients were studied; 35 patients with untreated chronic gastric ulcer, 19 patients who had been treated medically, 28 who had been treated by vagotomy and pyloroplasty, and 13 treated by partial gastrectomy.

The incorporation of facilities for taking biopsy specimens under vision through a fibreoptic gastroscope was a new development when the present study began. The actual taking of the specimen requires some skill and experience. For these reasons, in the early stages, biopsy specimens were obtained from only two sites apart from the ulcer itself. As proficiency developed and the safety of this technique of gastroscopy with biopsy was demonstrated in over $\mathbf{4 0 0}$ examinations without complication, it was decided to obtain biopsy specimens from four standard sites. The great majority of the patients included in the present series were examined in this way. 


\section{Methods}

\section{GASTROSCOPY WITH BIOPSY}

At gastroscopy, a methodical survey of the stomach was made. On gastroscopic criteria the gastric mucosa was judged to be normal or to show superficial or atrophic gastritis. When the gastritis appeared to be zonal or patchy, a description of its distribution within the stomach was recorded. If a gastric ulcer was seen, photographs were taken and then cytological and multiple biopsy specimens were obtained from the ulcer margin to exclude malignancy (Gear, Truelove, and Williams, 1969).

Biopsy specimens were then obtained from four standard sites (Fig. 1). These were: (1) the prepyloric region; (2) the middle of the lesser curve just proximal to the incisura; (3) high on the lesser curve about $1 \frac{1}{2}$ inches distal to the cardia, and (4) on the middle of the greater surve at a point opposite the site of the mid-lesser curve biopsy.

Usually two specimens were obtained from each site to ensure having adequate tissue for histological examination.

In order to view the fundal area fully, the patient was turned into the right lateral position.

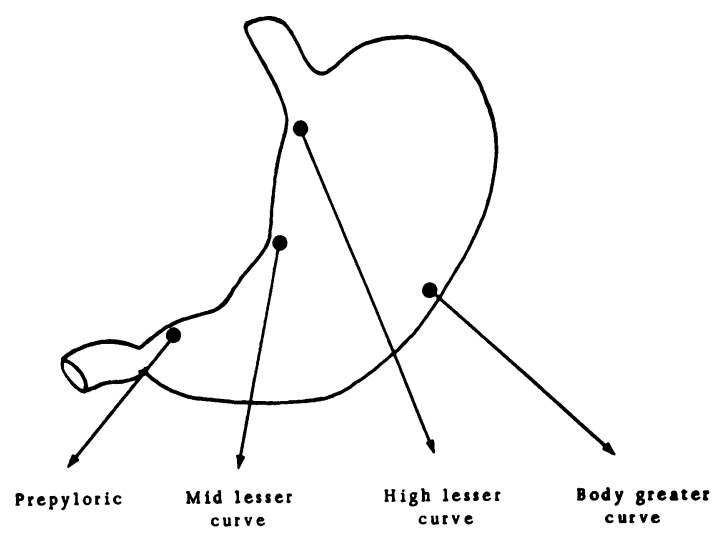

Fig. 1 The four standard biopsy sites

\section{HISTOLOGY}

The gastric biopsy specimens from the ulcer edge and the four standard sites were handled according to the methods described by Whitehead, Gear, and Truelove (1971).

The histological preparations of all the gastric biopsy specimens (over 500 in all) were examined by one of us (R.W.) and graded according to the scale outlined below. The report was filed in the patient's ase notes in the usual way. Some time later all the biopsy specimens were examined again, on this occasion 'blind', the pathologist being unaware of the name of the patient, the type of treatment, and the site of the biopsy. The results of the two separate gradings for each biopsy correlated almost perfectly. The result of the second examination was taken as the more reliable, as on this occasion all the biopsy specimens were examined consecutively over a period of a few days.

TYPES OF MUCOSAL APPEARANCE RECORDED Normal gastric mucosa

The mucosa was taken to be normal when none of the changes described below were seen.

\section{Chronic superficial gastritis}

In this grade there is a plasma cell and lymphocyte infiltrate in the superficial layer of the gastric mucosa. Eosinophils and neutrophils in small numbers are also seen. There are no histological changes in the epithelium of the glands, but the superficial epithelium and the epithelium lining the pits frequently shows evidence of damage. There is often a loss of PAS-positivity, the cells appearing flattened and basophilic and their nuclei somewhat pleomorphic, pyknotic, and palisaded. Occasionally, patches of intestinal metaplasia were seen in a mucosa which otherwise showed no signs of atrophic changes.

\section{Chronic active superficial gastritis}

This shows similar appearances to those graded as 'chronic superficial gastritis', but the cellular infiltrate is denser and includes a high percentage of polymorphonuclear cells. These acute inflammatory cells are seen invading and apparently 'destroying' the surface epithelium and the epithelium lining a proportion of the gastric pits.

\section{Chronic atrophic gastritis}

When fully developed the gastric mucosa is decreased in thickness and there is a chronic inflammatory cell infiltrate involving the whole thickness of the lamina propria. The superficial epithelium may show changes similar to those in chronic superficial gastritis. There is also atrophy of glands, whether these are of body or pyloric type. Sometimes the specialized glands of the body mucosa are replaced by shorter simple tubes lined by a low columnar epithelium of a simple mucussecreting type (pseudopyloric metaplasia).

\section{Mild, severe, and moderate atrophic gastritis}

Mild atrophic gastritis was recorded when the atrophic changes were slight. Severe atrophic gastritis was recorded when virtually all the normal gland arrangement was lost. Moderate atrophic 
gastritis was recorded when the changes were intermediate.

\section{Intestinal metaplasia}

Intestinal metaplasia was recorded quite independently of the degree of gastritis. The epithelium is of intestinal type with a brush border, and includes goblet cells and sometimes Paneth cells.

Although intestinal metaplasia is almost always associated with the changes of atrophic gastritis this is not invariable and this feature has therefore been considered separately.

In some biopsy specimens, mucosa was seen which was indistinguishable from the gastric mucosal atrophy seen in pernicious anaemia, with little or no inflammatory infiltrate and with complete intestinalization. However, as this was an uncommon finding, such specimens were classified as showing 'severe atrophic gastritis'.

Thus, in summary, three separate decisions were taken about each gastric biopsy: (1) the mucosal type-antral or body (in severe atrophic gastritis this was sometimes not possible); (2) the grade of gastritis; and (3) the presence or absence of intestinal metaplasia. The classification of gastritis is discussed in greater detail by Whitehead et al (1971).

\section{MEDICAL TREATMENT}

The patients in this group were treated conventionally with antacids (and carbenoxolone sodium in some cases) until the symptoms abated and improvement in the barium meal appearances occurred. A few patients in this group were taking part in a therapeutic trial of an antipepsin compound (Depepsin or SN263).

\section{SURGICAL TREATMENT}

\section{Vagotomy and pyloroplasty}

Most of the patients treated during this study were operated on by one of two surgeons (Mr B. L. Dowling or M.W.L.G.) using an identical technique as far as possible. The gastric ulcer was exposed, either through the pyloroplasty incision or through a separate gastrotomy, and wedge segments of the ulcer edge were excised and sent for immediate frozen section histology in order to confirm the benign nature of the lesion. Either a selective or a truncal vagotomy was then performed, the choice of procedure being determined randomly. A Heineke-Mikulicz pyloroplasty was always performed. The operation was completed by closing the pyloroplasty in two layers, using catgut sutures. Postoperative management was conventional, the patient usually being allowed home on a normal diet 10-14 days after operation.

The patients treated four or more years earlier had been operated on by a variety of surgeons, but in every case a vagotomy and pyloroplasty had been performed for gastric ulcer.

\section{Partial gastrectomy}

The operation varied somewhat in this group, but in all except two patients, a Billroth I gastrectomy was performed with removal of about half the stomach and gastroduodenal anastomosis. In some cases a vagotomy was added. In two patients, a more extensive resection necessitated a Polya type of gastrojejunal anastomosis.

\section{Results}

GASTRITIS IN UNTREATED GASTRIC ULCER

The 35 patients with untreated gastric ulcer consisted of 23 males and 12 females, whose age ranged from 26 years to 81 years, with an average of 55 years. The length of history varied widely from a few weeks to many years.

\section{Findings at gastroscopy}

The ulcer was well seen in all patients in this series and was judged to be benign in appearance. The size of the ulcer was judged roughly by using the biopsy forceps or cytology brush as a scale at the side of the crater when viewed through the gastroscope. Most prepyloric ulcers were small, about 0.5 to $1 \mathrm{~cm}$ in diameter. Ulcers in the body of the stomach were much more variable in size, ranging from $1 \mathrm{~cm}$ up to large craters 3 to $4 \mathrm{~cm}$ in diameter.

Most of the patients with ulcers in the body of the stomach showed the changes of superficial or atrophic gastritis (or both) at gastroscopy, while patients with prepyloric ulcers often had normal mucosa in the proximal half of the gastric mucosa.

The question of the correlation between the gastroscopic appearances of gastritis and the histological changes in the biopsy specimens is discussed in more detail below.

\section{Histological findings}

Twenty-eight $(80 \%)$ of the 35 patients studied had one or more biopsy specimens showing atrophic changes in some degree. The other seven patients all had at least one biopsy showing superficial gastritis, so no patient in the series had an entirely normal gastric mucosa.

Only two $(6 \%)$ patients of the 35 studied had the same grade of gastritis in all four biopsy specimens. In the remainder, there was wide variation from one biopsy to another, with a tendency for the more severe atrophic changes to be found distally in the antrum and on the lesser curve, while the greater curve was least affected. 
Intestinal metaplasia was most commonly seen in biopsy specimens with severe atrophic gastritis, but four examples of metaplasia occurred in association with superficial gastritis. In general, the distribution of metaplasia in the stomach followed that of moderate and severe atrophic gastritis.

Fifty-four per cent of prepyloric and $31 \%$ of midlesser curve biopsy specimens showed metaplasia, while only $8.5 \%$ of high lesser curve and $11 \%$ of greater curve biopsies showed the changes.

There were 14 prepyloric (or antral) ulcers, and 21 ulcers in the body of the stomach in the group of 35 patients studied. There was a marked difference in the distribution and severity of the gastritis seen with these two sites of gastric ulcer. In general, the changes seen were more severe and more extensive in ulcers of the body of the stomach. Thus, $21 \%$ of biopsy specimens were normal in patients with prepyloric ulcers, whereas only $2 \%$ were normal in the group of body ulcers. Only $30 \%$ of biopsy specimens from patients with prepyloric ulcers showed atrophic changes, while $50 \%$ were found in patients with ulcers in the body of the stomach and the atrophic changes were often severe.

Only $13 \%$ of biopsy specimens in patients with prepyloric ulcer showed metaplasia, while $36 \%$ in patients with ulcers in the body of the stomach showed the change.

Men were more liable to show severe gastritis than women, despite the fact that there was a greater proportion of prepyloric ulcers in men. Men had a greater percentage of biopsy specimens showing atrophic changes, and also almost double the proportion showing chronic active superficial gastritis. The difference is even more striking when intestinal metaplasia is considered. This change was twice as frequent in biopsy specimens from men as in those from women.

In view of many previous published reports, it was somewhat surprising to find that age seemed to have no effect on the distribution or severity of gastritis in the group of patients with untreated gastric ulcer. There were 17 patients aged 55 or less and 18 aged over 55 . In these two groups the percentages of biopsy specimens showing atrophic gastritis, superficial gastritis, and intestinal metaplasia were virtually identical.

However, the two groups are not entirely comparable, as the group over 55 included a disproportionate number of women, with less severe gastritis.

The series of 35 patients studied was arbitrarily divided into two groups: 16 with a short history of two years or less and 19 with a long history of more than two years.

There were twice as many patients with ulcers in the body of the stomach in the group with long his- tories. The patients with long histories tended to have a greater number of abnormal biopsy specimens at all grades of gastritis than those with a short history.

Intestinal metaplasia was more than twice as frequent in biopsies from patients with long histories than those with short histories.

\section{GASTRITIS IN MEDICALLY TREATED GASTRIC ULCER \\ Clinical features and findings at gastroscopy}

Over the three-year period of the study, 28 patients who were seen with a gastric ulcer had a gastroscopy before and after medical treatment. For the reasons discussed earlier, only two biopsy specimens were obtained at most of the early gastroscopies. Those patients from whom four biopsy specimens were obtained are included in the appropriate untreated or healed series as well as in the group studied in this section. The period between the two gastroscopic examinations was never less than three months, and often extended to 18 months or two years.

One patient aged 42 , with a greater curve ulcer, died some months after the first gastroscopy of an unrecognized perforation of the ulcer with generalized peritonitis. He had severe bronchitis and emphysema and was admitted in respiratory failure. The perforation was discovered only at postmortem examination.

Of the remaining 27 patients, eight were found to have a persistent or recurrent gastric ulcer at the time of the second gastroscopy. However, in two of the eight unhealed ulcers the appearances suggested partial healing. Two patients had a vagotomy and pyloroplasty later because of persistence of symptoms and thus are included in the surgical series.

In patients with a healed ulcer of the body of the stomach, it was common to find persisting gastroscopic evidence of atrophic gastritis. By contrast, in the patients with a healed prepyloric ulcer, the mucosa often looked relatively normal except in the prepyloric region.

\section{Histological findings}

In most cases, the biopsy specimens obtained at the second examination showed a similar, or worse, grade of gastritis than those from the first examination. In a few instances an improvement occurred with reversion to normal from superficial gastritis, or to superficial gastritis from mild atrophic changes. On the other hand, patients with severe atrophic gastritis at the first examination never showed improvement at the second examination.

In the medically treated group, the distribution and severity of the gastritic changes at the four standard biopsy sites were similar to those seen in 
unhealed gastric ulcer, with a slightly worse picture in the two proximal biopsy sites. In the majority of patients there was no improvement in the gastritis when the ulcer had healed. The distribution of intestinal metaplasia was also similar to that found in untreated gastric ulcer.

\section{VAGOTOMY AND PYLOROPLASTY}

Clinical features and gastroscopic findings

Thirty-seven patients were studied, nine at four years or more after the operation and 28 with a short follow up of three to six months.

The nine patients who had been studied for more than four years were part of a series of 45 patients who had a vagotomy and pyloroplasty for gastric ulcer between 1962 and 1965. All the nine patients studied were symptomless, except one who was mildly anaemic and suffered occasional attacks of dyspepsia. At gastroscopy no sign of an ulcer was found but two patients had a slight degree of hourglass narrowing at the site of the previous ulcer. The appearance of the mucosa was very variable. In two patients it appeared virtually normal except in the immediate vicinity of the pyloroplasty.

Twenty-eight patients with gastric ulcer were treated by vagotomy and pyloroplasty during the three-year period of the study. The length of follow up varied from three years to a few weeks. All the ulcers healed promptly and no patient developed a recurrent ulcer. Nineteen of the 28 had a gastroscopic examination three months or more after surgery, as well as a preoperative gastroscopy.

\section{Histological findings}

Nineteen of the 28 patients treated by vagotomy and pyloroplasty during the period of this study had only two gastric biopsy specimens taken before and after operation. In 11 patients, four biopsy specimens were obtained at the second gastroscopy and these 11 are therefore also included in the four biopsy studies.

In general, the histological appearances remained the same or became somewhat worse after surgery, but in two cases improvement occurred, with reversion of a mild atrophic change to superficial inflammation.

There were 20 patients included in the four biopsy studies. In general, the mucosal changes appeared to be worse after surgery despite prompt healing of the ulcer. It is interesting to note that the greatest deterioration occurred in the two proximal biopsy sites, high on the lesser curve and on the greater curve, respectively. The most severe and extensive gastritis was found at the high lesser curve site, almost $90 \%$ of specimens showing moderate or severe atrophic changes. Severe atrophic gastritis was found equally commonly $(30 \%)$ at all four biopsy sites, in contrast to the decreasing frequency found in the proximal sites in untreated gastric ulcer.

Intestinal metaplasia was frequently found after surgical treatment. About $60 \%$ of biopsy specimens from the prepyloric and two lesser curve sites showed metaplasia, while $40 \%$ of greater curve specimens showed the change.

Severe atrophic gastritis was three times more frequent in patients with a long dyspeptic history before operation, but the overall incidence of all grades of atrophic change was not markedly different in the two groups. Intestinal metaplasia was more frequent in the group with a history of dyspepsia of more than two years.

One interesting finding was the occurrence of the chronic active form of superficial gastritis, especially in biopsy specimens taken from the greater curve. This histological appearance was found only in the patients with a short ulcer history who were reexamined early after operation. It was never seen in patients with a long ulcer history, or in those who had had their operation four years or more previously.

Atrophic gastritis was more frequent in biopsy specimens from the group examined four years or more after operation, but the difference from those followed for one year was not marked. The finding of biopsy specimens showing chronic active superficial gastritis was confined to the patients followed for less than a year. Intestinal metaplasia was found in $70 \%$ of biopsy specimens from the long-term group, but in only $50 \%$ from the short-term group.

In summary, the specimens from patients treated by vagotomy and pyloroplasty showed a high proportion of moderate or severe atrophic gastritis at all four biopsy sites and a high incidence of intestinal metaplasia. In those patients studied before and after surgery, the gastritis commonly became worse, even over a short period of observation.

It is revealing to compare the findings in the untreated group of patients with those observed in patients with the ulcer healed, whether treated medically or surgically by vagotomy and pyloroplasty. This part of the study is confined to patients examined by biopsy from all four standard sites.

As far as intestinal metaplasia is concerned, this change was just as frequent in patients whose gastric ulcer had healed with medical treatment as it was in the untreated patients, while it was twice as frequent in the patients whose gastric ulcer had healed after vagotomy and pyloroplasty.

PARTIAL GASTRECTOMY

Thirteen patients were studied, nine some years after 
operation and four within one year. The types of operation were Billroth I (7), Polya (2), and vagotomy and antrectomy (4). Eleven had suffered from chronic ulcers of the body of the stomach, the other two from prepyloric ulcers. On gastroscopy, evidence of severe atrophic gastritis was common. The biopsy specimens were obtained from three sites: mid lesser curve, high lesser curve, and greater curve. The specimens commonly showed atrophic gastritis evenly distributed over all three biopsy sites. In view of this, it is somewhat surprising that only two biopsy specimens $(6 \%)$ showed intestinal metaplasia.

CORRELATION BETWEEN GASTROSCOPIC AND HISTOLOGICAL FINDINGS

The appearance of the mucosa was recorded at the time of gastroscopy and was later correlated with the histological grade of gastritis. The results are set out in Table $\mathbf{I}$.

\begin{tabular}{|c|c|c|c|c|c|}
\hline \multirow{2}{*}{$\begin{array}{l}\text { Gastroscopic } \\
\text { Appearance }\end{array}$} & \multicolumn{4}{|c|}{ Histological Grade at Corresponding Sites } & \multirow[t]{2}{*}{ Total } \\
\hline & Normal & $\begin{array}{l}\text { Superficial } \\
\text { Gastritis }\end{array}$ & $\begin{array}{l}\text { Mixed } \\
\text { Superficial } \\
\text { and } \\
\text { Atrophic } \\
\text { Gastritis }\end{array}$ & $\begin{array}{l}\text { Atrophic } \\
\text { Gastritis }\end{array}$ & \\
\hline \multirow{3}{*}{$\begin{array}{l}\text { Normal } \\
\text { Superficial } \\
\text { gastritis } \\
\text { Mixed superficial } \\
\text { and atrophic } \\
\text { gastritis } \\
\text { Atrophic gastritis }\end{array}$} & 4 & 0 & $\mathbf{0}$ & 2 & 6 \\
\hline & 1 & 11 & $\mathbf{0}$ & 14 & 26 \\
\hline & 0 & 2 & 29 & 0 & 31 \\
\hline
\end{tabular}

Table I Correlation between gastroscopic appearances and histological findings

When the gastritis appeared at gastroscopy to be superficial in one part of the stomach and atrophic in another, it was classified as 'mixed'. As can be seen from Table I, most of these cases also had a 'mixed' gastritis on histology, having atrophic changes in one biopsy and chronic superficial gastritis in another. When the gastroscopy report mentioned one area particularly as atrophic, then the appropriate biopsy site was used in assessing the correlation between visual and histological appearances. It was unusual to find mucosa which looked normal and therefore was always noted. Those few cases where no mention of the state of the mucosa was made in the gastroscopy report were excluded from Table I. Thus 82 cases of the total of 87 with biopsy specimens from four sites were included.

It can be seen from Table I that when 'mixed' superficial and atrophic changes were seen at gastroscopy, in over $90 \%$ of cases the histological findings agreed. Atrophic gastritis was also correctly diag- nosed at gastroscopy in $\mathbf{9 0 \%}$ of cases. However, when superficial gastritis was diagnosed at gastroscopy, in half the cases atrophic gastritis was found. There were too few cases with a normal mucosa to make a satisfactory assessment of the reliability of the gastroscopic judgment.

Thus, the signs suggesting atrophic changes at gastroscopy are a reliable guide to the histological state of the mucosa. The signs suggesting superficial gastritis, however, often underestimate the severity of the gastritis. Contact bleeding is probably a fairly reliable guide to the presence of gastritis, but does not differentiate between superficial and atrophic gastritis.

\section{Discussion}

The present study has shown that the gastritis associated with chronic gastric ulcer is regional. The distal part of the stomach and mid lesser curve are the most severely affected both by atrophic change and by intestinal metaplasia. The distribution of metaplasia agrees well with the pattern found by Graham and Schade (1965) and Stemmermann and Hayashi (1968). These findings show that single biopsy specimens obtained blind from the greater curve area may miss large areas of atrophic mucosa or metaplasia in the rest of the stomach. We can confirm the finding of Joske et al (1955) and of other workers that two specimens obtained close together from the same area of the stomach usually show the same degree of gastritis, but specimens obtained from the greater curve and lesser curve may show wide variations.

Specimens from the body of the stomach on the greater curve side are least likely to show gastritic changes. Conversely, if the greater curve specimen shows severe atrophic gastritis then it is likely that the other sites are similarly affected.

Ulcers of the body of the stomach are associated with more extensive and more severe gastritis than ulcers of the prepyloric region. Prepyloric ulcers are associated with gastritis which is localised to the distal biopsy sites and is often superficial with little evidence of atrophic changes or metaplasia. The difference in gastritis between the two ulcer types persists after the ulcer has healed, whether treatment be medical or surgical.

The distribution of gastritic changes in the stomach after treatment is similar to that found in untreated gastric ulcer. Severe atrophic gastritis and intestinal metaplasia are found most frequently in prepyloric and mid-lesser curve biopsy sites, and tend to spread proximally in treated gastric ulcer, particularly after surgery. The overall frequency of severe changes was also increased in the treated 
group, although in a few cases, definite improvement occurred after healing of the ulcer.

There has been a great deal of controversy about the gastroscopic diagnosis of gastritis. The early gastroscopists like Schindler recognized three types: hypertrophic, superficial, and atrophic gastritis. Palmer (1954) felt that atrophic gastritis could be diagnosed reliably at gastroscopy, but that superficial gastritis and normal mucosa could not be distinguished in many cases. Joske et al (1955) went further and stated that gastroscopy was too unreliable to be of any value in the diagnosis of any grade of gastritis. However, they based their views on specimens obtained by blind biopsy techniques. The present study, with biopsy under direct vision, has confirmed Palmer's view that atrophic gastritis can be diagnosed reliably at gastroscopy.

In conclusion, the present study suggests that gastritis is the basic disease process and gastric ulceration is a secondary phenomenon. The gastritis is widespread in association with chronic peptic ulcer of the body of the stomach, whereas it is commonly more localized with chronic prepyloric ulcer. Superficial or atrophic gastritis has been found to persist or even worsen after healing of the ulcer, whether treatment be medical or surgical. This finding disposes of the idea that the gastritis associated with gastric ulcer is simply a zonal gastritis secondary to the ulcer.

We wish to thank the Nuffield Committee for making a research grant to one of us (M.W.L.G.).
References

Benedict, E. B. (1948). An operating gastroscope. Gastroenterology, $11,281-283$.

Faber, K. (1935). Gastritis and Its Consequences. Oxford University Press, London.

Gear, M. W. L., Truelove, S. C., Williams, D. G., Massarella, G. R., and Boddington, M. M. (1969). Gastric cancer simulating benign gastric ulcer. Brit. J. Surg., 56, 739-742.

Graham, R. I., and Schade, R. O. K. (1965). The distribution of intestinal metaplasia in macroscopic specimen, demonstrated by a histochemical method. Acta path. microbiol. scand., 65, 53-59.

Hancock, P. E. T., and Shiner, M. (1958). Combined gastroscopy and gastric biopsy under direct vision. Lancet, 1, 1204-1205.

Hebbel, R. (1943). Chronic gastritis: its relation to gastric and duodenal ulcer and to gastric carcinoma. Amer. J. Path., 19, 43-71.

Hebbel, R. (1949). The topography of chronic gastritis in otherwise normal stomachs. Amer. J. Path., 25, 125-141.

Joske, R. A., Finckh, E. S., and Wood, I. J. (1955). Gastric biopsy: a study of 1,000 consecutive successful gastric biopsies. Quart. J. Med., 24, 269-294.

Kenamore, B. (1940): A biopsy forceps for the flexible gastroscope. Amer. J. dig. Dis., 7, 539.

Macdonald, W. C., and Rubin, C. E. (1967). Gastric biopsy: a critical evaluation. Gastroenterology, 53, 143-170.

Magnus, H. A. (1946). The pathology of simple gastritis. J. Path. Bact., 58, 431-439.

Magnus, H. A. (1952). Gastritis. In Modern Trends in Gastro-enterology. 1st series, edited by F. H. Jones, pp. 323-351. Butterworth, London.

Palmer, E. D. (1954). Gastritis: A revaluation. Medicine (Baltimore), 33, 199-290.

Sanders, G. B., and Mecray, P. M. (1941). Pseudogastritis of operative origin. Ann. Surg., 114, 986-996.

Schindler, R. (1947). Gastritis. Heineman, London.

Schindler, R., Necheles, H., and Gold, R. (1939). Surgical gastritis: a study on the genesis of gastritis found in resected stomachs with particular reference to so-called 'antral gastritis' associated with ulcer. Surg. Gynec. Obstet., 69, 281-286.

Stemmermann, G. N., and Hayashi, T. (1968). Intestinal metaplasia of the gastric mucosa: a gross and microscopic study of its distribution in various disease states. J. nat. Cancer Inst., 41, 627-634.

Whitehead, R., Gear, M. W. L., and Truelove, S. C. (1971). The histological diagnosis of chronic gastritis in fibreoptic gastroscope biopsy specimens. J. clin. Path. In Press. 